\title{
Modeling and Simulation of Bacterial Outer Membranes with Lipopolysaccharides and Enterobacterial Common Antigen
}

\author{
Ya Gao ${ }^{1,2}$, Jumin Lee ${ }^{2}$, Göran Widmalm ${ }^{3^{*}}$, and Wonpil $\mathrm{Im}^{2,4^{*}}$ \\ ${ }^{1}$ School of Mathematics, Physics and Statistics, Shanghai University of Engineering Science, \\ Shanghai 201620, China \\ ${ }^{2}$ Department of Biological Sciences, Department of Chemistry, and Department of \\ Bioengineering, Lehigh University, Bethlehem, PA 18015, USA \\ ${ }^{3}$ Department of Organic Chemistry, Arrhenius Laboratory, Stockholm University, SE-10691 \\ Stockholm, Sweden \\ ${ }^{4}$ School of Computational Sciences, Korea Institute for Advanced Study, Seoul 02455, \\ Republic of Korea
}

*Corresponding author e-mail: Goran.Widmalm@su.se and wonpil@lehigh.edu 
Table S1. Molecular dynamics simulation system information.

\begin{tabular}{|c|c|c|c|c|c|c|}
\hline \multirow{2}{*}{ System names } & \multicolumn{5}{|c|}{ Number of glycoconjugates in each leaflet } & \multirow{2}{*}{ Initial size $\left(\AA^{3}\right)$} \\
\hline & LPS & $E C A_{L P S}$ & $E C_{P V P G}$ & $\mathrm{ECA}_{\mathrm{DPPG}}$ & ECA $_{P S P G}$ & \\
\hline${ }^{100}$ LPS (0159) & 100 & & & & & $138 \times 138 \times 340$ \\
\hline${ }^{95} \mathrm{LPS}^{5} \mathrm{ECA}_{\mathrm{LPS}}$ & 95 & 5 & & & & $138 \times 138 \times 426$ \\
\hline${ }^{95} \mathrm{LPS}^{5} \mathrm{ECA}_{\mathrm{PVPG}}$ & 95 & & 5 & & & $138 \times 138 \times 376$ \\
\hline${ }^{95} \mathrm{LPS}^{5} \mathrm{ECA}_{\mathrm{DPPG}}$ & 95 & & & 5 & & $138 \times 138 \times 376$ \\
\hline${ }^{95} \mathrm{LPS}^{5}{ }^{\mathrm{E} C A_{\mathrm{PSPG}}}$ & 95 & & & & 5 & $138 \times 138 \times 376$ \\
\hline${ }^{75} \mathrm{LPS}^{25} \mathrm{ECA}_{\mathrm{LPS}}$ & 72 & 24 & & & & $135 \times 135 \times 430$ \\
\hline${ }^{50} \mathrm{LPS}^{50} \mathrm{ECA}_{\mathrm{LPS}}$ & 48 & 48 & & & & $135 \times 135 \times 440$ \\
\hline${ }^{75} \mathrm{LPS}^{25} \mathrm{ECA}_{\mathrm{PG}}$ & 72 & & 8 & 8 & 8 & $135 \times 135 \times 378$ \\
\hline${ }^{50} \mathrm{LPS}^{25} \mathrm{ECA}_{\mathrm{LPS}}{ }^{25} \mathrm{ECA}_{\mathrm{PG}}$ & 48 & 24 & 8 & 8 & 8 & $135 \times 135 \times 440$ \\
\hline${ }^{50} \mathrm{LPS}^{50} \mathrm{ECA}_{\mathrm{PG}}$ & 48 & & 16 & 16 & 16 & $135 \times 135 \times 380$ \\
\hline
\end{tabular}


Table S2. Averaged glycosidic torsion angles $(\phi, \psi)$ (with standard deviations) of $\alpha$-L-Fucp$(1 \rightarrow 4)$-D-GlcpNAc and $\beta$-D-GlcpNAc-(1 $\rightarrow 3)$-D-GIcpNAc in 0159-PS (RU 2 to 4) for which the interatomic distance H5@Fuc (side-chain) and 05@GIcNAc (non-branched residue) is less than $2.7 \AA$.

\begin{tabular}{|c|c|c|c|}
\hline \multirow{2}{*}{ Systems } & $\alpha$ Fucp-(1 $\rightarrow 4)$-GlcpNAc & $\beta G \mid c p N A c-(1 \rightarrow 3)-$ GlcpNAc & Population \\
\hline & $\phi, \psi\left({ }^{\circ}\right)$ & $\phi, \psi\left(^{\circ}\right)$ & $(\%)$ \\
\hline${ }^{100}$ LPS & $46.4 \pm 10.8,22.5 \pm 8.1$ & $54.2 \pm 7.9,13.2 \pm 9.1$ & 41.7 \\
\hline${ }^{95} \mathrm{LPS}^{5} \mathrm{ECA}_{\text {LPS }}$ & $46.8 \pm 10.8,22.8 \pm 8.2$ & $52.6 \pm 8.5,13.5 \pm 9.5$ & 46.3 \\
\hline${ }^{95} \mathrm{LPS}^{5} \mathrm{ECA}_{\mathrm{PVPG}}$ & $46.5 \pm 10.8,22.7 \pm 8.2$ & $52.5 \pm 8.6,12.5 \pm 9.2$ & 30.6 \\
\hline${ }^{95} \mathrm{LPS}^{5} \mathrm{ECA}_{\mathrm{DPPG}}$ & $46.4 \pm 10.8,23.0 \pm 8.2$ & $51.9 \pm 9.0,12.4 \pm 9.2$ & 45.7 \\
\hline${ }^{95}$ LPS $^{5}{ }^{E C A}$ PSPG & $46.8 \pm 10.9,23.2 \pm 8.3$ & $50.5 \pm 9.2,13.4 \pm 9.6$ & 29.8 \\
\hline${ }^{75} \mathrm{LPS}^{25} \mathrm{ECA}_{\text {LPS }}$ & $46.9 \pm 10.9,22.5 \pm 8.2$ & $53.4 \pm 8.1,14.7 \pm 9.5$ & 41.7 \\
\hline${ }^{50}$ LPS $^{50}$ ECA $_{\text {LPS }}$ & $46.8 \pm 11.0,22.6 \pm 8.2$ & $52.7 \pm 8.4,15.0 \pm 9.8$ & 34.8 \\
\hline${ }^{75} \mathrm{LPS}^{25} \mathrm{ECA}_{\mathrm{PG}}$ & $46.4 \pm 10.8,23.0 \pm 8.2$ & $51.8 \pm 8.7,13.0 \pm 9.2$ & 46.0 \\
\hline${ }^{50} \mathrm{LPS}^{25} \mathrm{ECA}_{\mathrm{LPS}}{ }^{25} \mathrm{ECA}_{\mathrm{PG}}$ & $46.1 \pm 10.6,22.2 \pm 8.1$ & $53.9 \pm 7.9,12.3 \pm 8.7$ & 51.7 \\
\hline${ }^{50} \mathrm{LPS}^{50} \mathrm{ECA}_{\mathrm{PG}}$ & $45.5 \pm 10.8,22.8 \pm 8.2$ & $54.4 \pm 8.2,11.8 \pm 8.7$ & 31.8 \\
\hline
\end{tabular}




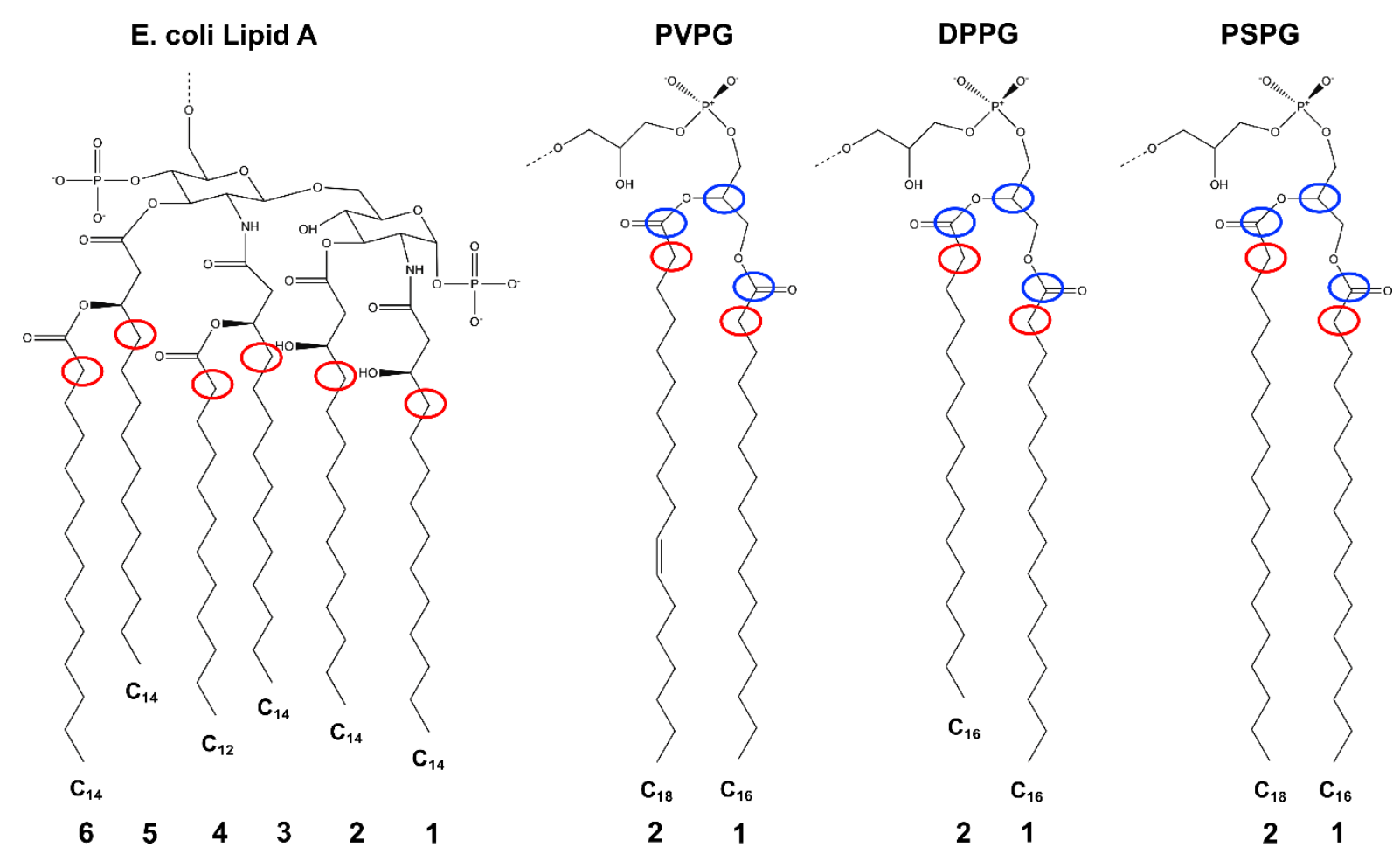

Figure S1. Chemical structures of E. coli lipid A, PVPG, DPPG, and PSPG. Carbons in the red ovals are used for the hydrophobic thickness calculation. Carbons in the red ovals in lipid A and those in the blue ovals in PVPG, DPPG, and PSPG are used for the Voronoi tessellation calculation. 


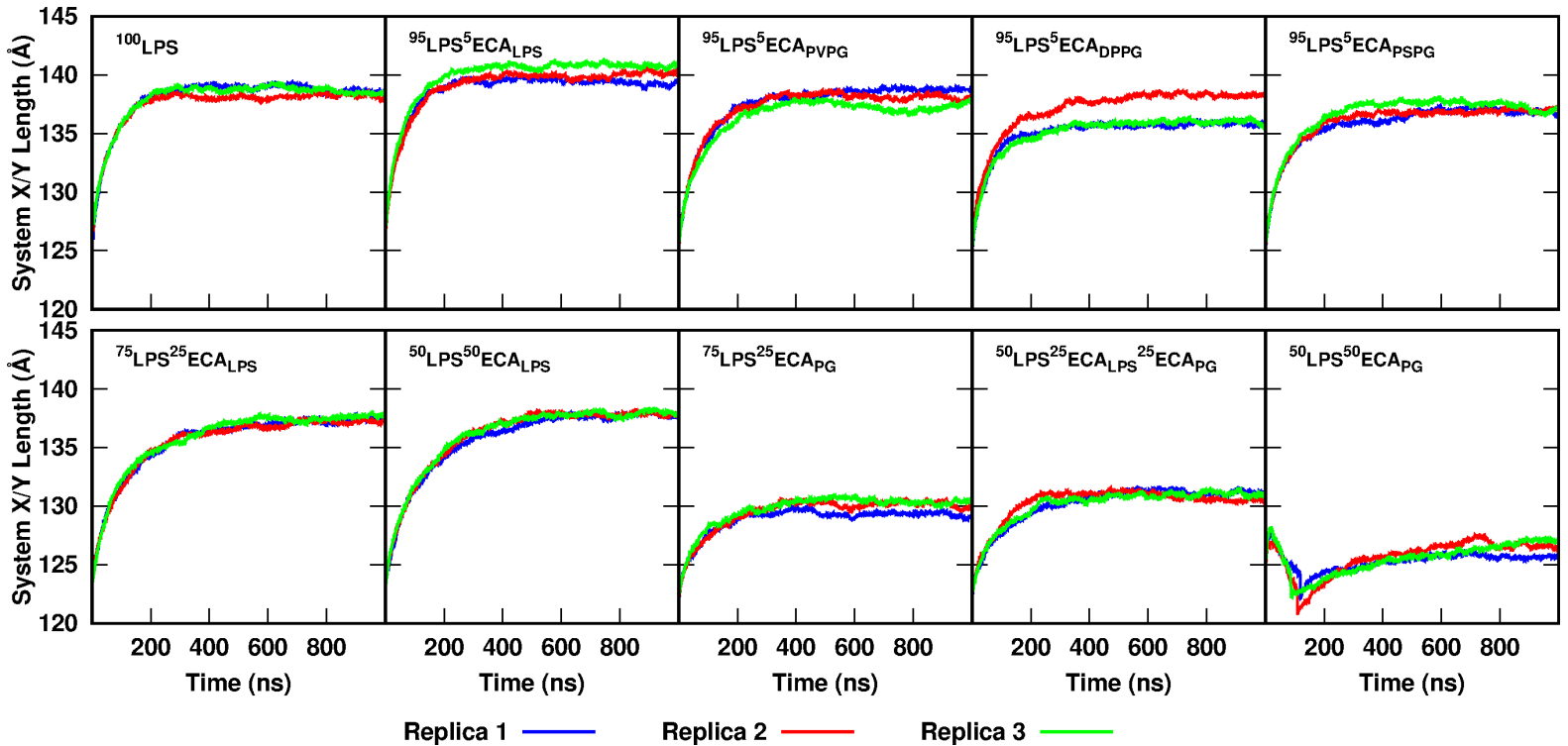

Figure S2. Time-series of $X$ or $Y$ system length. 

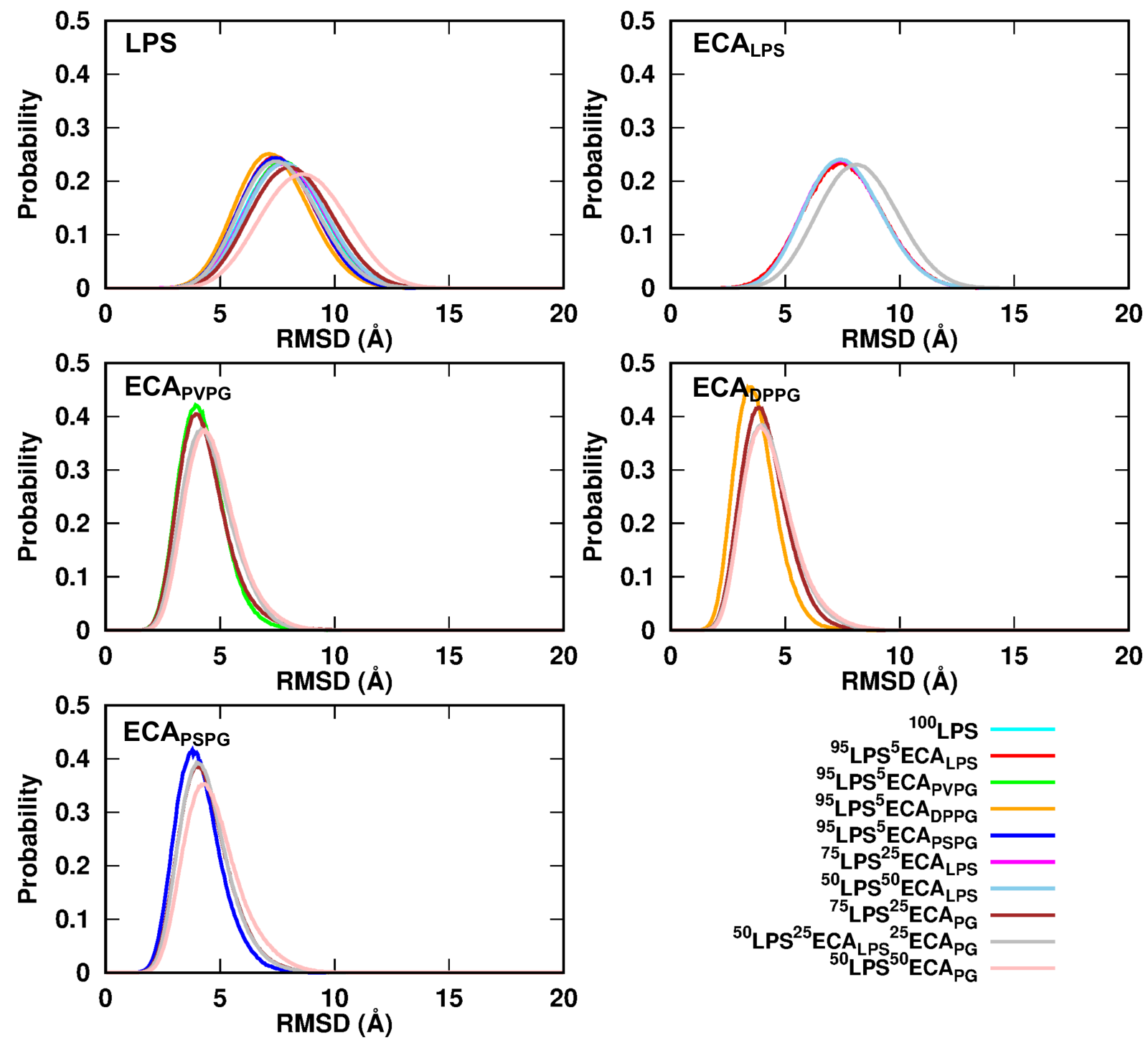

Figure S3. Pairwise RMSD distributions for residue 1 (i.e., lipid A or phosphatidylglycerol lipids) of each LPS and ECA. 

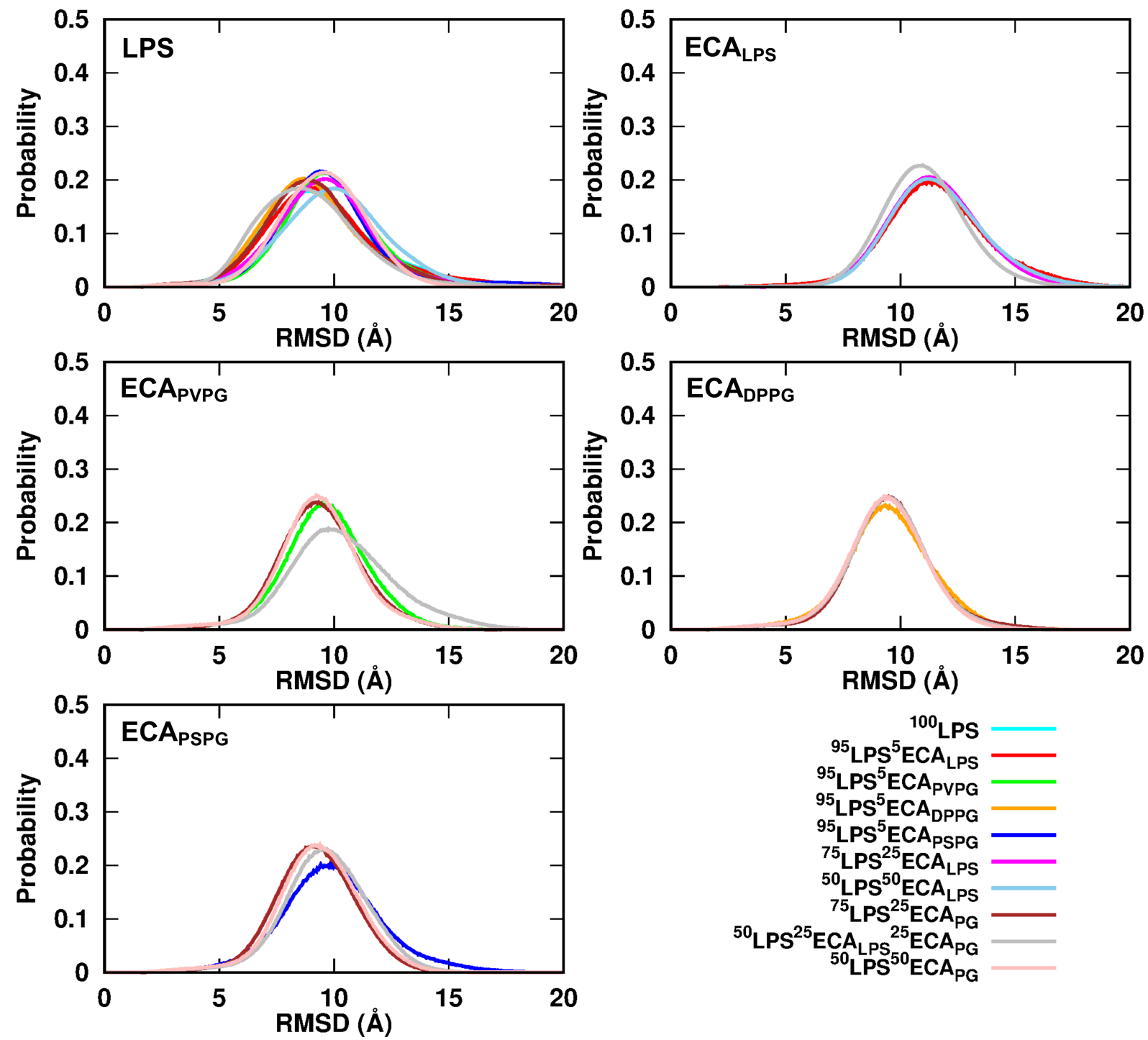

Figure S4. Pairwise RMSD distributions for all sugars (i.e., except for residue 1) of each LPS and ECA. 


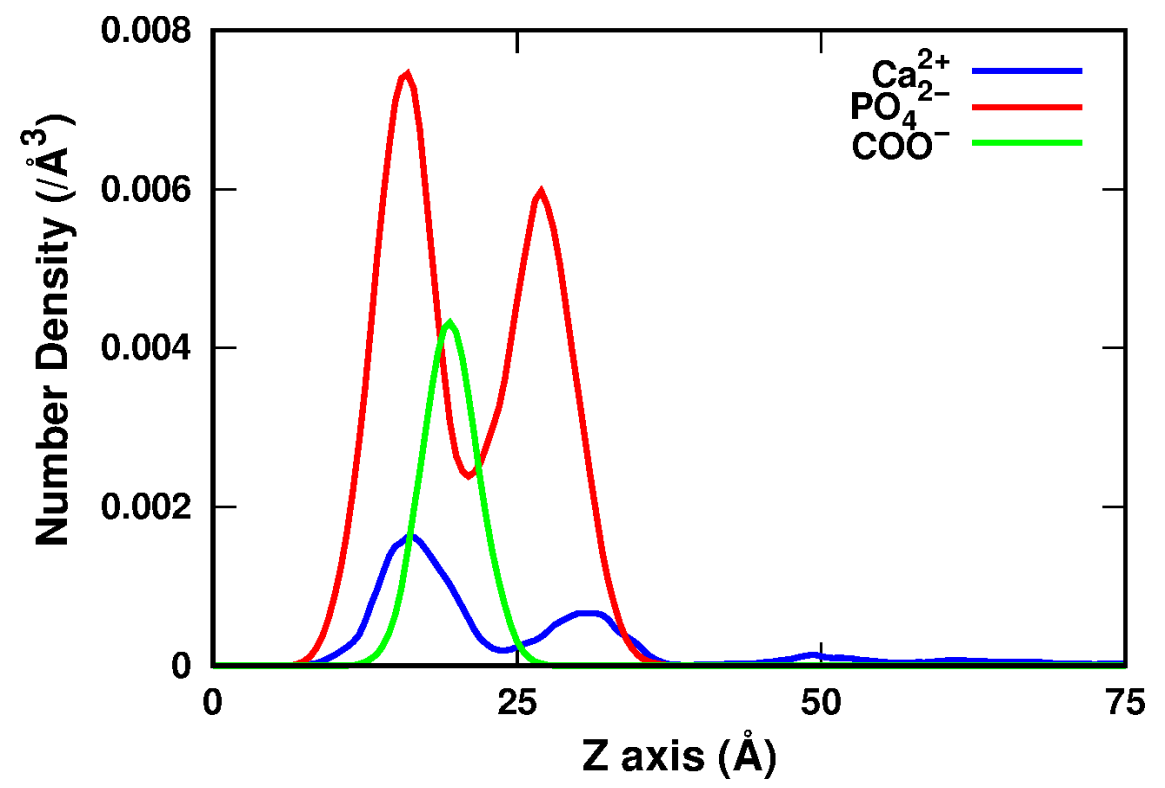

Figure S5. Distributions of ions and charged moieties of LPS $\left(\mathrm{Ca}^{2+}, \mathrm{PO}_{4}{ }^{2-}\right.$, and $\left.\mathrm{COO}^{-}\right)$along the Z-axis in system ${ }^{50} \mathrm{LPS}^{25} \mathrm{ECA}_{\mathrm{LPS}}{ }^{25} \mathrm{ECA} \mathrm{Ag}_{\mathrm{PG}}$. In the distributions, only the $Z>0$ membrane portion up to $Z=75 \AA$ is shown after symmetrization. 


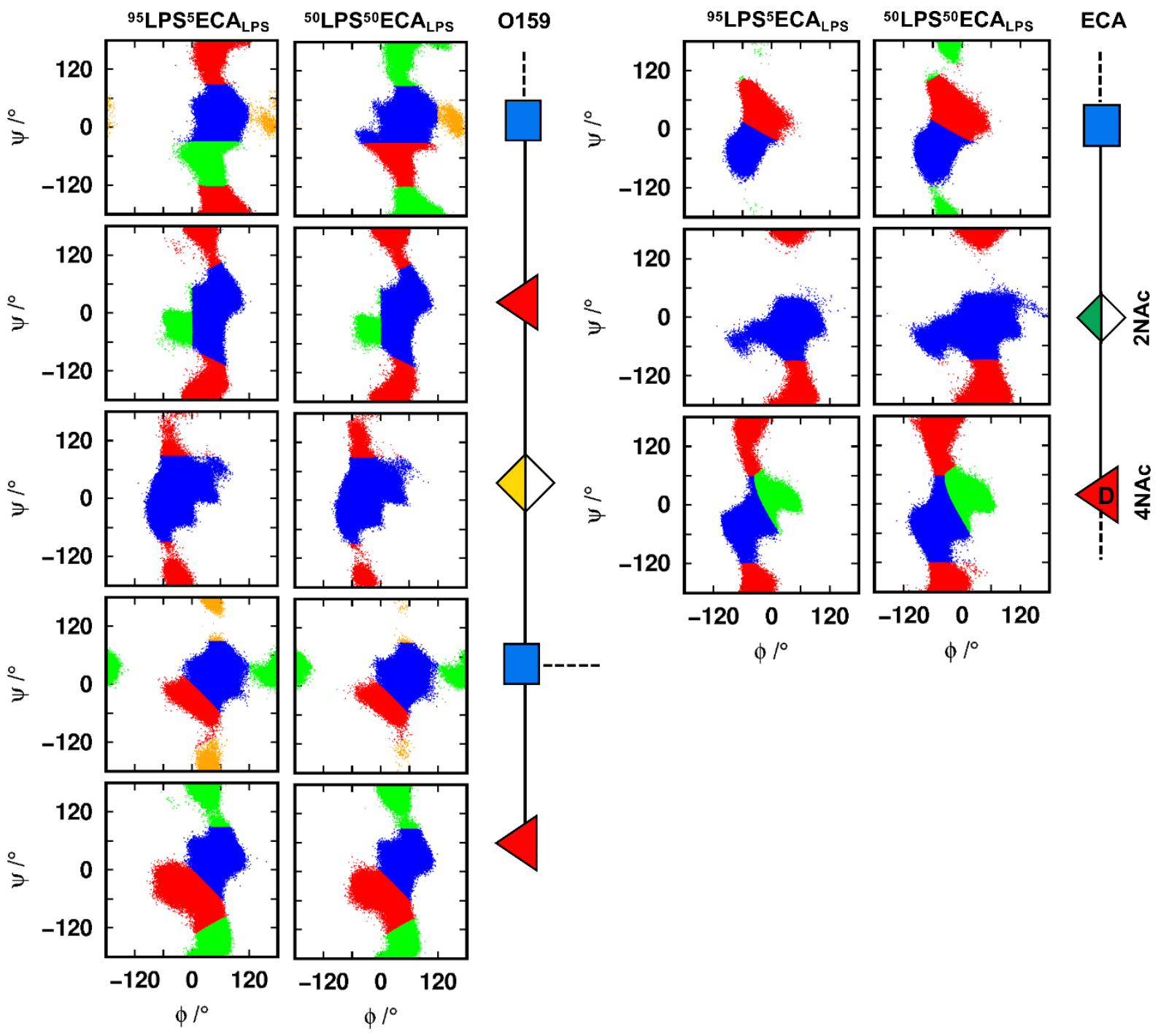

Figure S6. Glycosidic torsion angle distributions for each linkage of O159-PS and ECA-PS in systems ${ }^{95} \mathrm{LPS}^{5} \mathrm{ECA}_{\mathrm{LPS}}$ and ${ }^{50} \mathrm{LPS}^{50} \mathrm{ECA}_{\mathrm{LPS}}$. The distributions are clustered based on the glycosidic torsion angle: blue for the largest basin, red for the second largest basin, green for the third one, and orange for the fourth one. 


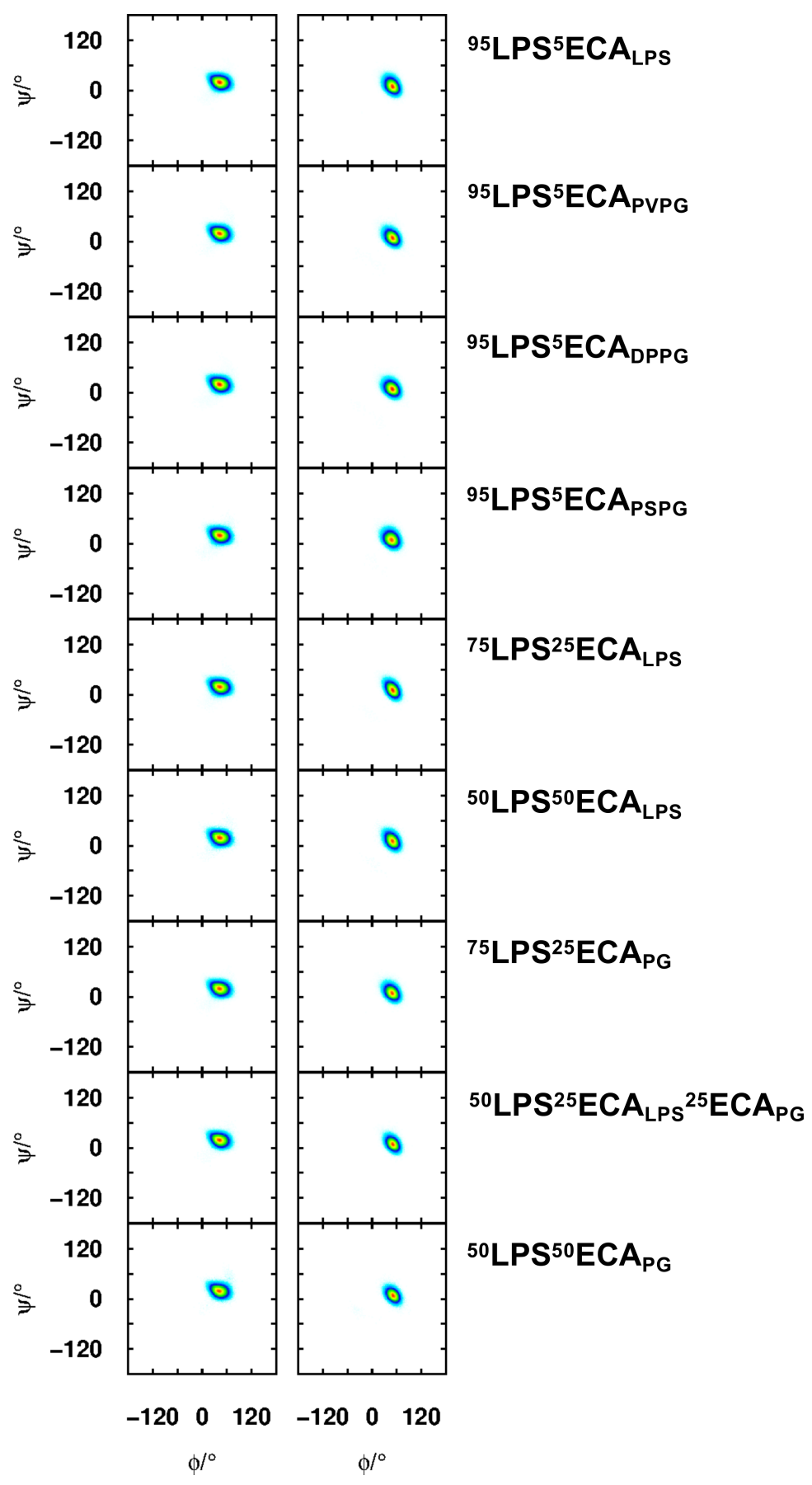

Figure S7. Two-dimensional distributions of glycosidic torsion angles $(\phi, \psi)$ of conformations of $\alpha$-L-Fucp-(1 $\rightarrow 4$ )-D-GlcpNAc (left) and $\beta$-D-GlcpNAc-(1 $\rightarrow 3)-D-G l c p N A c$ (right) in 0159-PS (RU 2 to 4) for which the interatomic distance H5@Fuc (side-chain) and O5@GlcNAc (nonbranched residue) is less than $2.7 \AA$. The density was rescaled by the maximum value: white for 0 , blue for 0.1 , green for 0.3 , yellow for 0.7 , and red for 1 . 